\title{
Relation between electric properties and water saturation for hematitic sandstone with frequency
}

\author{
Mohamed Mahmoud Gomaa \\ National Research Centre, Geophysical Sciences Dep., El-Tahrir St. Dokki, 12311, Egypt
}

\begin{abstract}
This paper focuses on the effect of water saturation on A. C. electrical conductivity and dielectric constant of fully and partially saturated hematitic sandstone sample (Aswan area, Egypt). The saturation of the sample was changed from partial to full saturation. Complex resistivity measurements at room temperature $\left(\sim 16^{\circ} \mathrm{C}\right)$, were performed in the frequency range from $0.1 \mathrm{~Hz}$ to $100 \mathrm{KHz}$. Experimental electrical spectra indicate, generally, that the electrical conductivity and dielectric constant vary strongly with water saturations and frequency. The low frequency electrical conductivity and dielectric constant are mainly controlled by surface conduction and polarization of the electrical double layer. The behaviour of the electrical conductivity and dielectric constant, with increasing water content, were argued to the orientational polarization of bound water for very low saturations, displacement of the excess surface charges for relatively low saturations, and free exchange of excess ions in double layer with the bulk electrolyte and generation of transient diffusion potentials which lag behind the applied field for high saturations.
\end{abstract}

Key words Electric properties - complex conductivity - frequency - hematite - water saturation

\section{Introduction}

Electrical spectra of porous rocks in the low frequency range reflect numerous polarization processes resulting mainly from rock heterogeneity. The first is the Maxwell-Wagner polarization (Mendelson and Cohen, 1982) due to differences in the electric bulk properties of rock components. Maxwell-Wagner theory allows the prediction of electrical spectra of mixtures from bulk partial volumes, the properties of their components and their microstructure. However, the Maxwell-Wagner theory was un-

Mailing address: Dr. Mohamed Mahmoud Gomaa, National Research Centre, Geophysical Sciences Dep., ElTahrir St. Dokki, 12311, Egypt.; e-mail: mmmsgomaa@ yahoo.com able to explain the anomalously large polarization in water-bearing rocks at low frequencies. The problem is that the mixture theory does not take into account surface conductivity and polarization, as well as clustering of components in disordered mixtures. The surface conductivity (in S) of a mineral grain is defined as the anomalous conductivity existing in the close vicinity of the mineral surface and integrated over the electrical double-layer thickness (Leroy and Revil, 2004). Surface conductivity is frequency dependent. Surface conductivity has two contributions; one is associated with the Stern layer and one with the diffuse layer in which counter-ion density obeys a Boltzmann distribution. None of these contributions can be neglected but the Stern layer contribution dominates.

This paper studies the A. C. electrical conductivity and dielectric constant of a partially and fully saturated hematitic sandstone sample (Aswan area, Egypt) in the frequency range from $0.1 \mathrm{~Hz}$ to $100 \mathrm{KHz}$. This geological mate- 
rial is an example of a multi-component system, a sand/hematite/water/air system. The concentration of the sample is mainly sand $(\sim 60 \%)$ with hematite $(\sim 40 \%)$. The grain size of sand in the sample is in the order of $\sim 60$ micrometer and the hematite is in the form of pigment. The porosity of the sample was calculated to be $(\sim 23 \%)$.

The aim of this work is to study the effect of water on the electrical conductivity and dielectric constant of the sample and to try to find out the main conduction mechanisms that are responsible for the measured behaviour. The sample was chosen as an example of the composite material that does not have a chemical reaction at the surface like clay, so the only supposed response may be argued only due to the addition of water. The study of the electrical properties of the sample may be of good importance in the application of spectral induced polarization in the field and in the interpretation of dielectric and conductivity spectroscopic measurements (A. C. electrical conductivity and dielectric constant).

The components of the wet hematitic sandstone interact strongly to affect the electrical behavior of the mixture. Dry rocks without a metallic component at room temperatures are good dielectrics with conductivity of the order of $10^{-10} \mathrm{~S} / \mathrm{m}$ and a relative dielectric constant of the order of several units. For partially and fully saturated rocks the water-rock interfaces give rise to large values of dielectric constant and strong frequency dependence (Chelidze and Guéguen, 1999; Chelidze et al., 1999) which can be explained by surface conduction and polarization processes. In the absence of an applied electric field, ions form a double layer at the water-rock interface. When an oscillating electric field is applied, the ions polarize around the rock grains giving rise to large dipoles and to large apparent dielectric constants (Chelidze and Guéguen, 1999; Gomaa, 2004). As the frequency increases, ions have less time to travel to polarize and contribute more to the conductivity, since they are more in phase with the applied oscillating electric field.

Saarenketo (1998) suggests that water in soils can be classified according to its electrical properties as:
1) Adsorption water that can be adsorbed by soil particle from the air. It consists of a monomolecular layer around negatively charged mineral surfaces and additional tightly and loosely bound adsorption water layers (Mitchell, 1992).

2) Capillary water: It is the moisture in soil which is not bound around grains, but does not respond to gravity.

3) Free water: is attracted to the soil solids so loosely that it may respond to the pull of gravity and move downwards.

The addition of a small amount of water causes dramatic increases in the values of dielectric constant and conductivity. The adsorbed liquid forms at first mono- or polylayer islands. As saturation increases, continuous fluid films are formed on the grain surface. If water molecules form strong bonds with the surface so that they cannot migrate in an electrical field, the most probable polarization mechanism is the orientation polarization of adsorbed water molecules or reversible hopping of $\mathrm{H}$ ions along the surface (Chelidze and Guéguen, 1999; Chelidze et al., 1999).

\section{Background on polarization phenomena of porous media}

The physical mechanism of the polarization of adsorbed layers can be either the orientational polarization of dipole molecules of water or the transfer of charges (ions in the Helmoholtz or diffuse double layer or protons) along hydroxilic groups or chains, so-called surface charge transfer complexes (Chelidze and Guéguen, 1999; Chelidze et al., 1999).

Pride (1994) referred to the mechanism of surface proton conduction, and he concluded that the hydrogen ions may efficiently conduct through the network of hydrogen-bonded water molecules present in the adsorbed layer, but he ignored this mechanism in his analysis.

The mechanism of polarization due to formation of a physically adsorbed multilayer of water is much more effective than the orientational polarization of dipoles and results in high experimental values of dielectric constant; which are larger than the largest dielectric con- 
stant of the mixture components (here hematite, sand, water or air). At low humidity, defect sites on grain surface present a high local charge density and a strong electrostatic field, that promote water dissociation providing protons as charge carriers of the hopping transport mechanism. Subsequent layers of water molecules are physically adsorbed. Charge transport occurs when $\left[\mathrm{H}_{3} \mathrm{O}\right]^{+}$releases a proton to a neighboring water molecule which accepts it while releasing another proton, and so forth (Grotthuss chain) (Garrouch, 2001; Garrouch and Sharma, 1994; Garcia-Belmonte et al., 2003). At high humidity, liquid water condenses in the pores, and electrolytic conduction takes place along with protonic transport in the adsorbed layers.

The contribution of surface processes to the ohmic conductivity of porous systems is considered by (Glover et al., 1994a; Pride, 1994; Dukhin, 1971; Glover et al., 1994b). Also, modeling of surface conductivity from the modeling of the electrical double layer combined with complexation of the surface sites is due to (Revil and Glover, 1998) for silica and Leroy and Revil (2004) for alumino-silicates. Revil and Glover (1998) give a value of the specific surface conduction associated with proton transfer in pure water to be $2.4 \times 10^{-9} \mathrm{~S}$ and examined the dependence of the surface conduction on salinity. Surface migration and diffusion evoke polarization mechanisms, which are often much stronger than the mixture component polarization (Chelidze and Guéguen, 1999; Chelidze et al., 1999).

Revil and Glover (1998) concluded that the contribution of the electrical diffuse layer to the surface conductivity can be neglected for silica and alumino-silicates, and that the main contribution to surface conductivity is probably due to counterions located in the Stern layer with surface mobilities approximately ten times smaller than in the bulk electrolyte. Another contribution is related to the proton transfer directly on the mineral surface and is salinity independent.

Schwarz (1962) proposed that at low frequencies the dielectric dispersion (for colloidal particles suspended in electrolytes) is due to the polarization of the counterion atmosphere around the particles by an electric field. The displacement of counter-ions in the double layer is shown to be equivalent to the existence of a surface capacitance displaying a diffusioncontrolled relaxation. This effect can be expressed by an additional apparent dielectric constant of the particles, exceeding their actual dielectric constant at low frequencies by many orders of magnitude. A distribution of the activation energies of the counter-ion particle movement on the particle surface is introduced to account for the relaxation spectrum of the dispersion curves.

Chelidze et al. (Chelidze and Guéguen, 1999; Chelidze et al., 1999) discussed the «gigantic low frequency polarization» theory (GLFP) caused by polarization of the electrical double layer (EDL) at the solid-liquid interface. The GLFP theory allows for a free exchange of EDL excess charges with the electrolyte (polarization). Application of an electric field distorts the EDL due to migration and diffusion of charges in it, which results in the appearance of local clouds with the excess of positive charges on one side of the particle and with their deficit on the other side within the bulk electrolyte, so concentration gradients near the particle appear, outside the EDL, in addition to the electric polarization field of the deformed EDL. However, steady-state polarization due to the induced concentration gradients is established controlled by diffusion over distances of the order of the linear dimension of the particle. The conduction currents lag behind the applied field, which appear as displacement currents and seen experimentally as high values of the dielectric constant. This results due to the large electric charge of a particle, and the large electrolyte conductivity.

Chew and Sen (1982) studied the dielectric constant and conductivity of a dilute immobile ensemble immersed in an electrolytic solution. They concluded that the existence of double layer greatly enhances the counter-ion current in the double layer region in the presence of an external field. This counter ion current piles up charges at the polar ends of the particle, which discharge into the bulk solution through a diffusion process in addition to a conduction process. The diffusion current in the diffusion cloud 
is out of phase with the applied field and has a pack up effect in the double layer causing the current to be out of phase. Hence the charges piled up at the ends of the sphere have an out of phase dipole moment. At high frequencies there is no build up of a large diffusion cloud outside the double layer.

Wong (1979) idealized a disseminated ore by a system of electronically conducting metallic spheres randomly dispersed in an electrolytic ally conducting host medium. With an applied electric field the transport of cations and anions in the inter-phase region near the metal-electrolyte interface will involve both drift and diffusion flux densities. The presence of excess or deficit makes ions to accumulate, which are loosely held to the surface. The cloud of ions surrounding each particle and the diffusion-controlled charge transfer reaction at the interface are responsible for inducing a time or frequency dependent electric dipole moment on each particle.

Mehaute and Crepy (1983) and (Glover et al., 1994a; Wong, 1987; Ruffet et al., 1991a, 1991b) considered the geometric effects resulting from the existence of fractal surfaces. They assumed that the internal surfaces of rocks are rough and examined how the charge layer is built up at the interface. When an electric field is applied along the Y-axis normal to the surface, ions have to migrate in this direction. As minerals are mainly dielectrics, ions do not penetrate into them and have to migrate along the rough surface in the $\mathrm{X}$-direction in order to progress further in the Y-direction. The main idea is that the exponent $\alpha$ of the Cole and Cole (1941) response function is closely related to the fractal dimension $D_{f}$ of the surface of the solid or, in other words, the distribution of relaxation times corresponds to the distribution of asperities on a rough surface. For these models, the frequency dependent exponent reflects the distribution of relaxation times, which in turn is connected with ion fluxes passing obstacles of various sizes on the rough surface. The experimental data of (Ruffet et al., 1991a, 1991b) has been made on the basis of the fractal surface model (Mehaute and Crepy, 1983; Wong, 1987).

The effect of rock/water interaction on the dielectric behavior of saturated sandstones has been shown in studies by Knight (Knight and Nur, 1987; Knight and Endres (1990). In these studies the real part of the dielectric constant of sandstone samples was measured as the level of water saturation was varied. The frequency range of the measurements was from $60 \mathrm{kHz}$ to $4 \mathrm{MHz}$. There was a general increase in the electrical conductivity and dielectric constant of the rock sample with the increase of water. They showed that there may be a critical saturation point for water wet samples, below which the conductivity increases gradually with saturation and above it the conductivity increase more slowly. They observed a power-law dependence of the dielectric constant $(\varepsilon)$ on the frequency $(w)$. They related the power law exponent to the water saturation and the surface area to volume ratio of grains. The power law response is thought to be due to the random nature of the constituents within the samples (Jonscher, 1999; Gomaa et al., 2000).

Chelidze et al. (Chelidze and Guéguen, 1999; Chelidze et al., 1999) gave an explanation for the large low-frequency polarization of the fully saturated rocks that it takes into account the heterogeneity in the distribution of the electrical charges on the mineral surfaces. In this model the relaxation time depends on the sizes of clusters of charges on the surfaces of minerals rather than on the grain size of particles.

\section{Experimental procedures}

Laboratory measurements were made on a thin disk-shaped hematitic sandstone sample with the following dimensions: thickness $6 \mathrm{~mm}$ and diameter $37 \mathrm{~mm}$. A two electrode technique was used with stainless electrodes (of Agilant dielectric test fixture 16451B) on the two opposite faces of the sample disk. Data were collected in the frequency range from $0.1 \mathrm{~Hz}$ up to $100 \mathrm{KHz}$ using a Hioki 3522- 50 LCR Hitester Impedance Analyzer. A voltage of $1 \mathrm{~V}$ was applied. The current density in the sample was nearly $1 \times 10^{-3}\left(\mathrm{microA} / \mathrm{cm}^{2}\right)$ at a frequency of $10 \mathrm{~Hz}$ for the saturated case and nearly $4 \times 10^{-6}$ $\left(\mathrm{microA} / \mathrm{cm}^{2}\right)$ at the same frequency for the dry case. 
The measured parameters are the series and parallel capacitance and the series and parallel resistance at different frequencies.

Measurement of the electrical properties of a material can be made in either the series or parallel mode. In the series mode, the complex impedance $\mathrm{Z}$ is measured, $Z=R_{s}-i X_{s}$, where $R_{s}$ is the series resistance (real impedance) and $X_{s}=1 / w C_{s}$ is the reactance, $C_{s}$ is the series capacitance. The complex resistivity is $\rho^{*}=Z \times(A / d)$, where $A$ is the cross-sectional area of the sample, $d$ is its thickness. The parameters were calculated from the following equations, $\operatorname{Re} Z=R_{s}, \operatorname{Im} Z=1 / w C_{s}$. For the parallel model $\sigma=1 / \rho, \quad \rho=R_{p} \times(A / d) . \quad \varepsilon^{\prime}=C p / C o$, $C o=(\mathrm{A} / \mathrm{d}) \varepsilon_{0}$, where $w$ is angular frequency, $R_{p}$ is the parallel resistance, $C_{p}$ is the parallel capacitance, $\varepsilon_{0}$ is the permittivity of free space $\left(8.85 \times 10^{-12} \mathrm{~F} / \mathrm{m}\right), G_{p}$ is the parallel conductance, and $C_{p}$ is the parallel capacitance.

The effect of water saturation on electrical conductivity is the most important parameter in this study. First, the sample was measured in the relative atmospheric humidity $(\sim 65 \%)$ in an isolated chamber (desiccators). The sample weight dry was $14.1 \mathrm{gm}$. The sample was fully saturated by initially evacuating it in a pressure vessel, then allowing distilled water to flow into the vessel. The saturated weight was 15.45 gm. Measurements on the fully saturated sample were made quickly after it was removed from the pressure vessel. Subsequent measurements, with the determination of weight, were made while the sample was let to dry. The bulk volume porosity of the interconnected pore volume of the $V_{p}$ sample was calculated as following:

1) The rock sample is weighed dry $\left(W_{1}\right)$;

2) The sample is then saturated with distilled water for 24 hours by using a vacuum pump; and

3) The completely saturated sample is weighed again $\left(\mathrm{W}_{2}\right)$ and the following equation is then applied:

$$
V_{p}=\left(W_{2}-W_{l}\right) / \sigma_{f}
$$

Where; $\sigma_{f}$ is the fluid density in $\mathrm{g} / \mathrm{cm}^{3} ; \sigma_{f}=1.0$ $\mathrm{g} / \mathrm{cm}^{3}$ for the distilled water.

The uncertainty associated with this method is of the order of $\pm 5 \%$. The relative saturation levels (in weight) were calculated as

$$
\mathrm{WS} \%=(\mathrm{SWW}-\mathrm{SWD}) /(\mathrm{SWS}-\mathrm{SWD}) \times 100
$$

The saturation level by volume is given by

$$
\mathrm{WV} \%=[(\mathrm{SWW}-\mathrm{SWD}) \times \mathrm{WVD} \times 100] /(\mathrm{SWW})
$$

where the WS\%= Relative water saturation percentage by weight, SWW= sample weight wet, $\mathrm{SWD}=$ sample weight dry, $\mathrm{SWS}=$ is the sample weight saturated, WV\% = Water volume percentage, and WVD = wet volume density.

\section{Interpretation and discussion of results}

The conductivity and dielectric constant increase with the increase of relative saturation level. Figures 1 and 2 show the variation of the dielectric constant and conductivity, respectively, with frequency at different levels of relative saturation. If the sample is dry it is supposed that there is no water and the pores are completely filled with air. As the saturation increases the adsorbed water forms the first monolayer around the grain surfaces and as the saturation increases gradually the adsorbed water tends to make another layer and so on until it makes multi-layers of water. The first monolayers are supposed to be bound to the surface of the grains and the others are supposed to be looser. The properties of adsorbed water differ significantly from those of the bulk liquid. This means that the properties of adsorbed molecules are intermediate between bulk water and ice (Chelidze and Guéguen, 1999; Chelidze et al., 1999; Knight and Nur, 1987; Knight and Endres, 1990; Hoekstra and Doyle, 1971). As the water saturation increases the adsorbed water coats completely the surfaces of the grains and begins to contact with each other forming islands of water between grains but those islands are still isolated from each other. The inner layers of water molecules form strong bonds with the surface so that they cannot migrate in an electrical field, and the most probable polarization mechanism is the orientation polarization of adsorbed water molecules or reversible hopping of $\mathrm{H}$ ions along the surface (Chelidze and Guéguen, 1999; Chelidze et al., 1999; Glover et al., 1994a). As saturation in- 


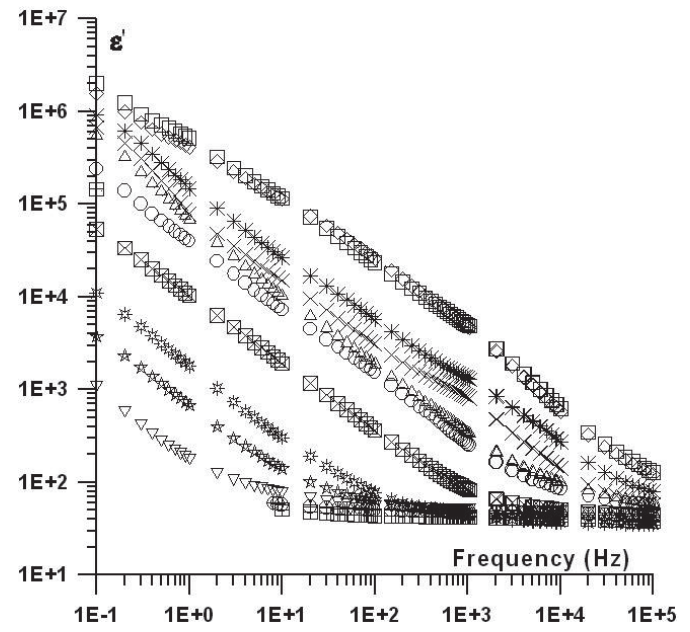

Fig. 1. The variation of the dielectric constant with frequency at different levels of relative saturations (in volume). $4 \% 0,8 \% 0,11 \% 0,14 \% 0,18 \% 0,25 \%$ $0,32 \% 0,40 \% 0,50 \% 0,55 \% 0,96 \% 0,100 \% 0$, respectively. There are many symbols that are not present.

creases, the electrodes become connected with continuous fluid film.

Figure 1 shows the variation of the dielectric constant with frequency at different levels of humidity and saturations. Values of the dielectric constant at low frequency $(0.1 \mathrm{~Hz})$ vary from $10^{3}$ for low saturated hematitic sandstone to $2 \times 10^{6}$ for full saturation, while the values at high frequency $\left(10^{5} \mathrm{~Hz}\right)$ range from 30 for low saturated hematitic sandstone to 200 for full saturation (nearly the order of water dielectric constant). The dielectric constant dispersion (fig. 1) can be generally divided into two regions with power law dependencies on frequency $\varepsilon \propto f^{-\alpha}$ with different exponents (slopes on the curves), which are not clearly separated at a certain frequency. In the first region at low frequencies the slope is steep $(\approx-0.67)$ for high saturation while, for relatively lower saturations it reaches a slope of value $\approx-0.5$ for low saturations. That region ends at nearly $2 \mathrm{~Hz}$ for $\sim 1 \%$ saturations, and extends for higher saturations to $20 \mathrm{KHz}$ (for $50 \%$ saturation), while it takes the whole frequency range for higher saturations. For the fully saturated sample

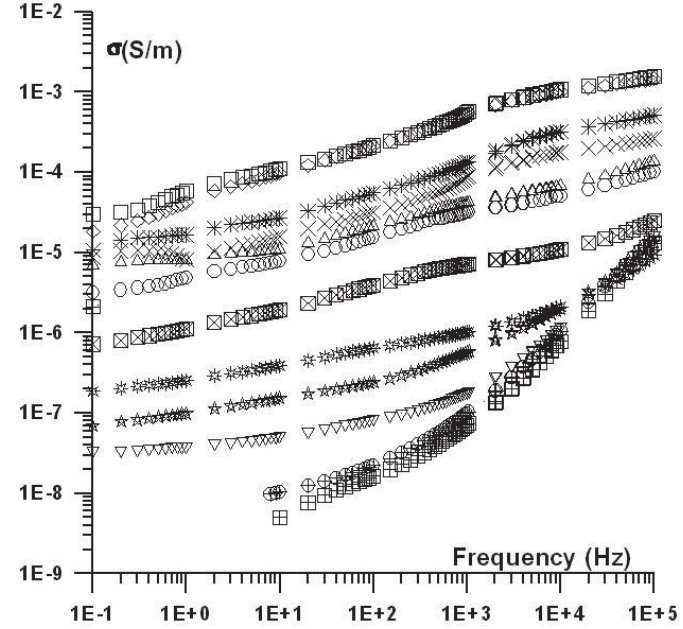

Fig. 2. The variation of the conductivity with frequency at different levels of relative saturations (in volume). $4 \% 0,8 \% 0,11 \% 0,14 \% 0,18 \% 0,25 \% 0$, $32 \% 0,40 \% 0,50 \% 0,55 \% 0,96 \% 0,100 \% 0$, respectively. There are many symbols that are not present.

nearly there is only one region. The high frequency region (region 2) has low values of the dielectric constant and nearly has no dispersion in dielectric constant for low saturations (below $11 \%$ ), while there is a slight dispersion for the dielectric constant with a very minute slope $(\approx-0.1)$ for high saturations (above $11 \%$ ).

At low saturations (mainly region 2) the physical mechanism of the polarization in adsorbed layers can be either the orientational polarization of dipole molecules of water, which may be effective at higher frequencies, or the transfer of charges (ions in the Helmoholtz or diffuse double layer or protons) along hydroxilic groups or chains, so-called surface charge transfer complexes (Chelidze and Guéguen, 1999; Chelidze et al., 1999) or surface polarization in the Stern layer (Schwan et al., 1962; Schwarz, 1962).

The gradual increase in the dielectric constant with the increase of humidity may be attributed to the decrease of the air space distances between the wet grains that behave as capacitors (for low saturations). The capacitance gradually increases as the humidity in- 
creases. When the adsorbed water begins to form a continuous path between the electrodes additional mechanisms are involved to contribute to increase the dielectric constant, namely the gigantic low frequency polarization described in the introduction (Chelidze et al., 1999; Chelidze, Guéguen, Ruffet, 1999). The continuous increase of the dielectric constant with decreasing the frequency may be attributed to the presence of a distribution of relaxation times due to the presence of polarization centers of different dimensions on the grains surfaces.

The frequency separating regions 1 and 2 increases with increasing the water saturation above the critical value (Wilkinson et al., 1983). Such critical relative water saturation is low (about 15\%) for water coating grains (Dukhin, 1971).

Charges either diffuse along the humidity coating the grains if it is unable to leave the surface, or diffuse through the bulk of the specimen. The data in the region of saturation is a result of the rock/water interaction. Charge transport can occur either through the bulk of the solid matrix (hematite) or along the grain boundaries of solid aggregates (water). When soil minerals are exposed to water, exchangeable ions go into solution, forming an ionic halo around the particles. Most of the ionic or covalent bonded rock forming minerals such as quartz, mica, and feldspars are nonconductors. When the surfaces of these minerals come into contact with liquid water, electrolytes are formed and ionic drift associated with the electrical field causes electrical conduction (Knight and Nur, 1987).

In the conductivity curves (fig. 2), two nearly corresponding regions may be generally found with power law variation of the conductivity with frequency, which are also clearly identified with two regions with different slopes. In the first region at low frequencies, for low saturations, there is slight dispersion for the conductivity. That region ends at nearly $100 \mathrm{~Hz}$ for saturations below $10 \%$ and extends for higher saturations to $20 \mathrm{KHz}$ (for $25 \%$ saturation), while it takes the whole frequency range for higher saturations. The second region has large slope $(\sim 0.9)$ for low saturation (below 10 $\%)$. Values of the conductivity at low frequency
$(0.1 \mathrm{~Hz})$ vary from $4 \times 10^{-9} \mathrm{Sm}^{-1}$ for low saturated hematitic sandstone to $3 \times 10^{-5} \mathrm{Sm}^{-1}$ for full saturation, while values for high frequency $\left(10^{5} \mathrm{~Hz}\right)$ ranges from $6 \times 10^{-6} \mathrm{Sm}^{-1}$ for low saturated hematitic sandstone to $2 \times 10^{-3} \mathrm{Sm}^{-1}$ for fully saturation (near the distilled water conductivity).

The first region is characterized by the presence of continuous paths of water and hematite between the electrodes (above about $10 \%$ saturation), thus causing nearly constant conductivity with frequency. Below this critical saturation (region 2), the continuous paths of water between the grains increase with increasing water content, thus the conductivity increases.

The low frequency conductivity (fig. 2) and dielectric constant change more than five decades with the change of saturation.

It should be noted that these curves are not completely identical in behaviour to other samples of the same material. Unlike curves may be attributed to the variation of the texture from sample to another (Parkhomenko, 1967; Efros and Shklovskii, 1976; Knight, 1983; Olhoeft, 1985; Sen, 1989; Levitskaya and Sternberg, 2000).

The equivalent circuit shown in fig. 3 is assumed to represent the sample in the humid case. The conducting grains are completely insulated (blocked) from each other by air. Here, $C_{\mathrm{g}}$ and $R_{\infty}$ is the capacitance and resistance of the sample at high frequencies, respectively, where no space charges accumulate. $C_{\mathrm{i}}$ is the interfacial capacitance (frequency independent) and $R_{\mathrm{i}}$ is the interfacial resistance (Macdonald, 1974). The resistance decreases as the frequency increases, at frequencies lower than the radio frequency range, where space charges are accumulated at interfaces.

When continuous paths are formed (due to water and/or hematite) in the sample, the impedance behavior is represented by the equivalent circuit shown in fig. 4 . Here $R_{\mathrm{D}}$ represents a direct frequency-independent conducting path between the two electrodes which is effective in the low frequency range (Gomaa, 1996). The additional resistor $R_{D}$ is equivalent to the formed percolation or continuous paths (due to water or hematite) in the sample. 


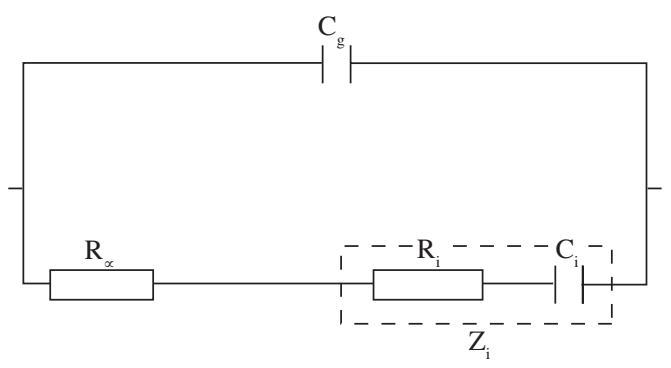

Fig. 3. Equivalent circuit that represent the sample in the low saturation case $\left(C_{\mathrm{g}}\right.$ and $R_{\infty}$ are the capacitance and resistance of the sample at the high frequency, respectively, $C_{\mathrm{i}}$ is the interfacial capacitance and $R_{\mathrm{i}}$ is the interfacial resistance).

Figures 5, 6, 7, 8 and 9 show the complex resistivity plane representation of the sample for partial and fully saturated cases. The data are presented in 5 figures due to the wide range of values on a linear scale. For the low partial saturation case nearly a straight line can be identified fig. 5. As the saturation increases an arc of a depressed semicircle is obtained in figs $6,7,8$ and 9 , which reflects the effect of the resistance $R_{\mathrm{D}}$. The depression of the arc in-

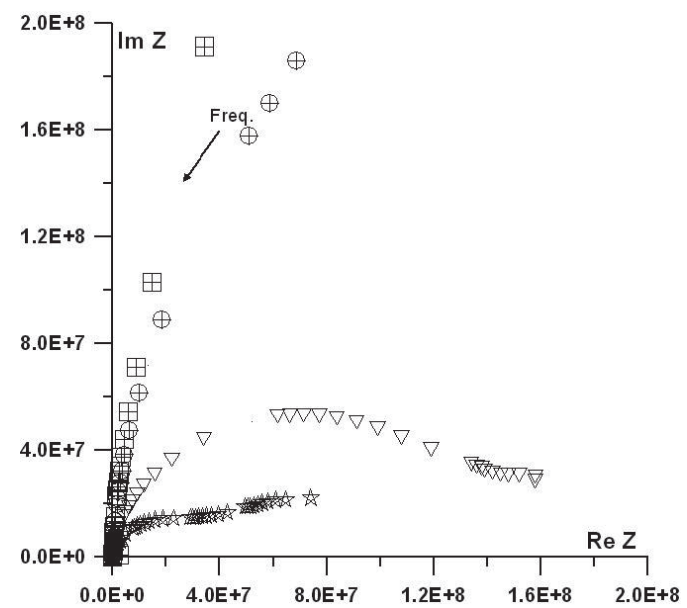

Fig. 5. The complex resistivity plane representation of the sample for relative saturations $4 \%(\boxplus), 8 \%$ $(\oplus), 11 \%(\nabla), 14 \%(\$)$, respectively.

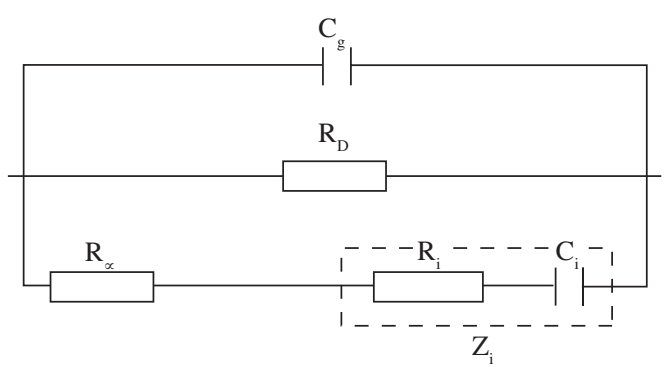

Fig. 4. Equivalent circuit that represents the sample in the high and full saturation case $\left(C_{\mathrm{g}}, R_{\propto}, C_{\mathrm{i}}\right.$ and $R_{\mathrm{i}}$ is the same as shown in fig. 3 ). $R_{\mathrm{D}}$ represents a frequency-independent conducting path between the two electrodes.

creases with the increase of the saturation, i.e. the conductivity increase (Grant, 1958). Such dominance of the real resistivity over the imaginary resistivity due to the increase of water saturation is representative to the increase in continuous conductor (hematite or water) paths in the sample.

Figure 9 can be divided into two parts, a high frequency part and a low frequency part. At higher frequencies the experimental points

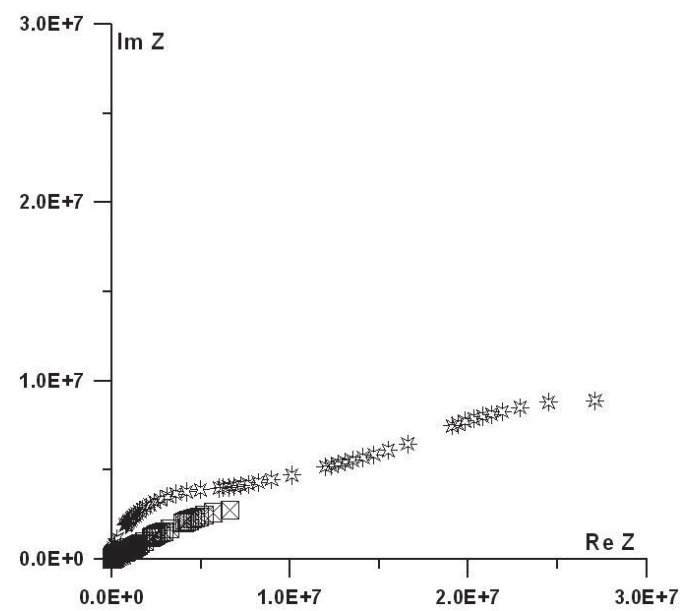

Fig. 6. The complex resistivity plane representation of the sample for relative saturations $18 \%$ (类), $25 \%$ $(\bigotimes)$, respectively. 


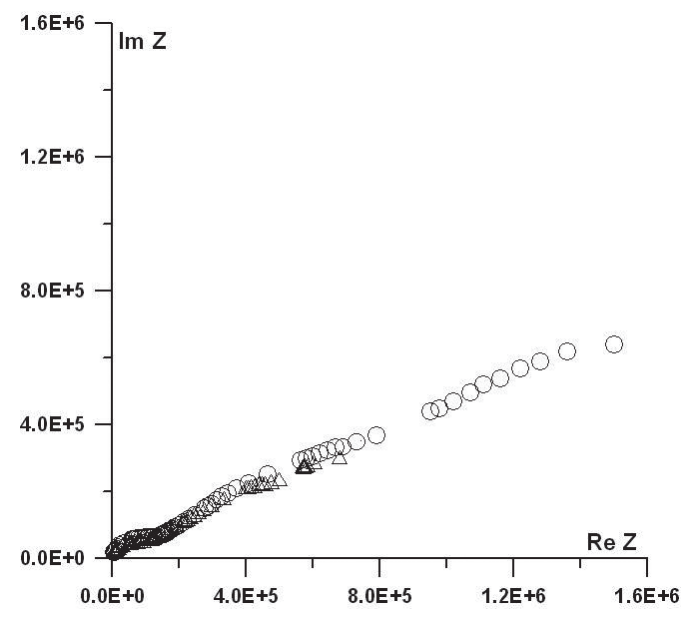

Fig. 7. The complex resistivity plane representation of the sample for relative saturations $32 \%(\bigcirc), 40 \%$ $(\triangle)$, respectively.

lie on an arc of a semicircle. The low frequency part is a straight line that reflects diffusion controlled resistivity. At low frequencies the electrode polarization takes place, which is humidity or saturation dependent. The straight line may represent diffusion controlled resis-

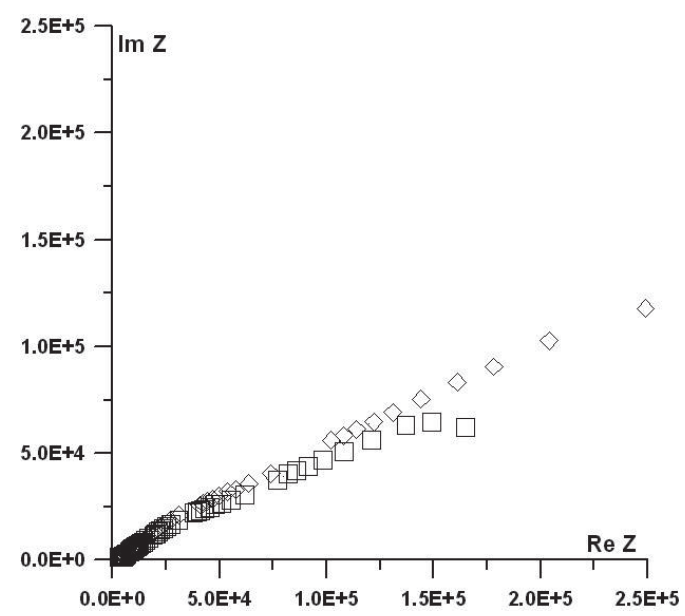

Fig. 9. The complex resistivity plane representation of the sample for relative saturations $96 \%(\diamond)$, $100 \%(\square)$, respectively.

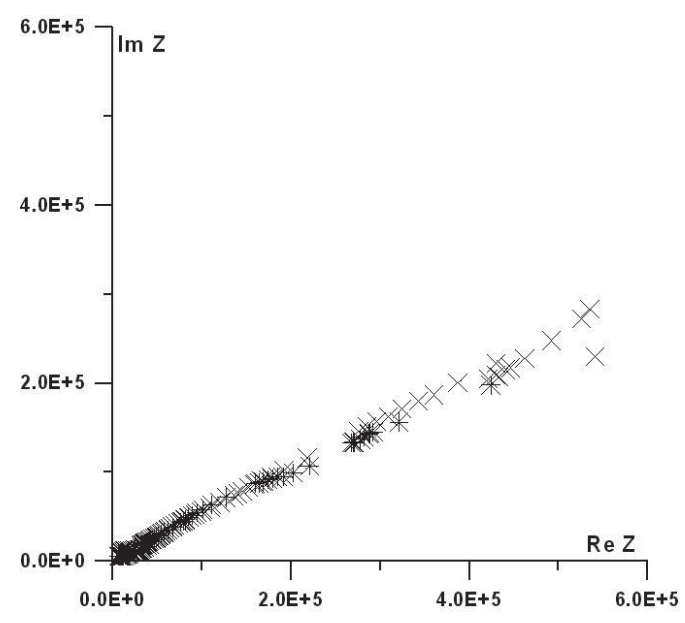

Fig. 8. The complex resistivity plane representation of the sample for relative saturations $50 \%(X), 55 \%$ (*), respectively.

tivity or Warburg impedance (Macdonald, 1974).

The Warburg impedance behavior at the low frequencies was attributed to the electrode effect by (Roberts and Lin, 1997); and also by (Knight and Nur, 1987). The latter authors stated that the frequency range of electrode effect extends to higher frequencies with the increase of water.

\section{Conclusions}

Effects of water saturation on the electrical conductivity and dielectric constant of humid, partially, and fully saturated hematitic sandstone sample is investigated in the frequency range from $0.1 \mathrm{~Hz}$ to $100 \mathrm{KHz}$. We change the saturation of the sample from normal relative humidity $(\sim 50 \% \mathrm{RH})$ to partially and fully saturated condition. Experimental data indicate that the electrical conductivity and dielectric constant vary strongly with water saturations. A transition from nearly normal to gigantic transport is observed at a certain frequency depending on the water content. The rate of change of dielectric constant with saturation is found to decrease with frequency. 
The changes in the electrical conductivity and dielectric constant due to humidity variation were argued to the mobile surface charges at watergrain interfaces. The simplest surface effect is due to surface migration and diffusion of charges under an applied field limited by a thin surface layer; this can be a conductive film or a closed electrical double layer. Film polarization is especially strong at moderate partial saturations, where it can produce strong LF polarization.

Very strong dielectric effects are caused by the polarization of «open» electric double layers on the solid-liquid interface at large saturations. Whereas polarization of bound charges in «closed» double layers produces relatively weak polarization, akin to that of films, diffusion potentials generated by the free exchange of ions between «open» deformed EDL and the adjoining electrolyte produce out-of-phase currents, and hence large values of effective dielectric constant. This model is known as GLFP; it can also be applied to heterogeneously charged surfaces.

The anomalous dielectric properties of partially saturated rocks are also interpreted partially using percolation theory. This theory predicts that, when the conductive fraction (water) increases, clustering of conductive inclusions develops, and the thickness of insulating gaps (air) between conductive clusters decreases, causing a large increment in the capacitance of the sample and its conductivity.

The results can be explained by electronic hopping conduction in the dry case, proton conduction in the humid case and low saturations, and electrolytic conduction along with protonic transport in the fully saturated case.

As a recommendation for this paper more controlled saturated samples should be measured to study clearly the effect of grain size and grain shape on the electrical properties of rock samples.

\section{REFERENCES}

Chelidze, T. and Y. GuÉGuen (1999): Electrical spectroscopy of porous rocks: a review - I. Theoretical models, Geophys. J. Int., 137, 1-15.

Chelidze, T., Y. GUÉGUEN and C. RufFet (1999): Electrical spectroscopy of porous rocks: a review -II. Experimental results and interpretation, Geophys. J. Int., 137, 16-34.
Chew, W.C., and P.N. SeN (1982): Dielectric enhancement due to electrochemical double layer : thin double layer approximation, J. Chem. Phys., 77 (9), 4683- 4693.

Cole, K.S. and R.H. Cole (1941): Dispersion and adsorption in dielectrics (I), J. Chem. Phys., 137, 341-351.

DukHIN, S.S. (1971): Dielectric properties of disperse systems, in «Surface and colloid science», (Matijevic, E., Editor), Wiley and sons, New York, Vol. 3, pp. 83- 166.

EFros, A.L. and B.I. SHKLOVSKII (1976): Critical behaviour of conductivity and dielectric constant near the metalnon-metal transition threshold, Phys. Status Sol., 137, 475-489.

Garcia-Belmonte, G., V. Kytin, T. Dittrich and BisQUERT (2003): Effect of humidity on the ac conductivity of nanoporous $\mathrm{TiO}_{2}, J$. Appl. Phys., 94 (8), 52615264.

GARROUCH, A.A. (2001): Effect of wettability and water saturation on the dielectric constant of hydrocarbons rocks, $41^{\text {st }}$ Annual Logging Symp. (SPWLA), paper NN.

Garrouch, A.A. and M.M. Sharma (1994): The influence of clay content, salinity, stress and wettability on the dielectric properties of brine-saturated rocks: $10 \mathrm{~Hz}$ to $10 \mathrm{MHz}$, Geophysics, 137, 909-917.

Glover, P.W.J., P.G. MEREDITH, P.R. SAMMONDS and S.A.F. MURREL (1994a): Ionic surface electrical conductivity in sandstone, J. Geophys. Res., 99, B11, 21635-21650.

GLOVER, P.W.J., P.G. MEREDITH, P.R. SAMMONDS and S.A.F. MuRREL (1994b): Measurements of complex electrical conductivity and fluid permeabilities in porous rocks at raised confining pressures, in Rock Mechanics in Petroleum Engineering, Proc. EUROROCK94, 29-36, Balkema, Amsterdam.

GomaA, M.M. (1996): Frequency response study on iron ore bearing rock samples, (M. Sc. Thesis, Cairo University, Egypt).

GomaA, M.M. (2004): Induced polarization Study on iron ore bearing rock samples, $(\mathrm{Ph}$. D. Thesis, Cairo University, Egypt).

GomaA, M.M., S.A. Hussain, E.A. El- Diwany, A.E. Bayoumi and M. GHobashy (2000): Modeling of A. C. electrical properties of humid sand and the effect of water content, $70^{\text {th }}$ annual international meeting, Society of Exploration Geophysics (SEG) and international Exposition, Session «Rock properties/Borehole: Rock Physics 1», Oral RPB6.7, Calgary, Alberta, Canada, 6-11 August, 1850-1853.

GRANT, F.A. (1958): Use of complex conductivity in the representation of dielectric phenomena, J. Appl. Phys., 29, $1,76-80$

Hoekstra, P. and W.T. Doyle (1971): Dielectric relaxation of surface adsorbed water, J. Colloid Interface Sci., 137, 513-521.

JONSCHER, A.K. (1999): Dielectric relaxation in solids, $J$. Phys. D: Appl. Phys., 32, R57-R70.

KNIGHT, R. (1983): The use of complex plane plots in studying the electrical response of rocks, J. Geomag. Geoelectr., 137, 767-776.

KNIGHT, R. J. and A. NuR (1987): The dielectric constant of sandstones, $50 \mathrm{kHz}$ to $4 \mathrm{MHz}$, Geophysics, 52, 644654.

Knight, R.J. and A.L. Endres (1990): A new concept in modeling the dielectric response of sandstones: Defin- 
ing a wetted rock and bulk water system, Geophysics, 55, 586-594.

Leroy, P., and A. ReVIL (2004): A triple layer model of the surface electrochemical properties of clay minerals, Journal of Colloid and Interface Science, 270, 2, 371380.

Levitskaya, T.M. and B.K. Sternberg (2000): Application of lumped-circuit method to studying soils at frequencies from $1 \mathrm{kHz}$ to $1 \mathrm{GHz}$., Radio Science, $\mathbf{3 5}$, 2, 371-383.

Macdonald, J.R. (1974): Binary electrolyte small-signal frequency response, Electroanal. Chem., Interfac. Electrochem., 53, 1-55.

Mehaute, A. and G. CREPY (1983): Introduction to transfer and motion in fractal media; the geometry of kinetics, Solid State Ionics, 137, 17-30.

Mendelson, K. S. and M.H. CoHen (1982): The effect of grain anisotropy on the electrical properties of sedimentary rocks, Geophys., 47 (2), 257-263.

MinOR, M., H.P. VAN LEEUWEN and J. LyKLEMA (1998): Low-Frequency Dielectric Response of Polystyrene Latex Dispersions, Journal of Colloid and Interface Science, 206 (2), 397-406.

Mitchell, J.K. (1992): Fundamentals of Soil Behaviour, $2^{\text {nd }}$ edn., (Wiley, New York), p. 437.

OlHOEFT, G.R. (1985): Low frequency electrical properties, Geophysics, 137, 2492-2503.

PARKHomenKo, E.I. (1967): Electrical Properties of Rocks, (Plenum Press, New York), p. 314.

PRIDE, S. (1994): Governing equations for the coupled electromagnetics and acoustics of porous media, Phys. Rev. B., 50, 1, 15678-15696.

Revil, A. and P.W.J. Glover (1998): Theory of ionic surface electrical conduction in porous media, Physical Review B., 55 (3) 1757-1773.

RoberTs, J. and W. Lin (1997): Electrical properties of partially saturated topopah spring tuff: water distribution as a function of saturation, Water Resources Research, 33, 577-587.
Ruffet, C., Y. GuÉGuen and M. DARot (1991a): Complex measurements and fractal nature of porosity, Geophysics, 137, 758-768.

Ruffet, C., Y. GuÉGUEN and M. DAROT (1991b): Rock conductivity and fractal nature of porosity, Terra Nova, 137, 265-275.

SAARENKETO, T. (1998): Electrical properties of water in clay and silty soils, Journal of Applied Geophysics, 40, 73-88.

Schwan, H.P., G. Schwarz, J. Maczuk and H. Pauly (1962): On the low-frequency dielectric dispersion of colloidal particles in electrolyte solution, Journal of Physical Chemistry, 66, 2626-2635.

ScHWARZ, G. (1962): A theory of the low-frequency dielectric dispersion of colloidal particles in electrolyte solution, Journal of Physical Chemistry, 66, 2636-2642.

SEN, P.N. (1989): Unified models of conductivity and membrane potential of porous media, Phys. Rev. B., 137, 9508-9517.

Shilov, V.N., A.V. Delgado, F. GonZalez-Caballero and C. Grosse (2001): Thin double layer theory of the wide-frequency range dielectric dispersion of suspensions of non-conducting spherical particles including surface conductivity of the stagnant layer, Colloids and Surfaces A: Physicochemical and Engineering Aspects, 192, 253-265.

Wilkinson, D., J.S. LANGER and P.N. SEN (1983): Enhancement of the dielectric constant near a percolation threshold, Phys. Rev. B, 28, 2, 1081-1087.

WONG, J. (1979): An electrochemical model of the inducedpolarization phenomenon in disseminated sulfide ores, Geophysics, 44, 1245-1265.

Wong, P.Z. (1987): Fractal surfaces in porous media, in Physics and Chemistry of Porous Media, 137, edited by J.P. Bahavar, J. Koplik and K.W. Winkler, Am. Inst. Phys., 154, 304-318.

(received May 7, 2008; accepted June 30, 2008) 